\title{
Erratum
}

\section{Brainstem cavernous malformations: the role of Gamma Knife surgery}

\author{
Clinical article
}

To THE EDITOR: We recently responded to the Letter to the Editor by Dr. Da Li and Dr. Jun-Ting Zhang, ${ }^{1}$ who commented on our paper on treatment of cavernous malformations (CMs) by Gamma Knife surgery (GKS) (Lee CC, Pan DHC, Chung WY, et al: Brainstem cavernous malformations: the role of Gamma Knife surgery. Clinical article. J Neurosurg 117 Suppl:164-169, December 2012). Drs. Li and Zhang pointed out that the methods we had used to calculate hemorrhage rates were dissimilar to those of other authors and suggested a different method to calculate hemorrhage rates after GKS. In reviewing our article, we found that the problem began with the way in which we had calculated patient-years during the initial 2 years of follow-up.

Originally we calculated patient-years in the initial 2 years post-GKS as totaling $90(45$ patients $\times 2$ years $=$ 90 patient-years). However, not all 45 patients were followed up for 2 full years; 16 patients were followed up for shorter periods. We therefore would like to take this opportunity to change our calculation on patient-years for the initial 2 years to the following:

Initial 2 years post-GKS: 70.0 patient-years $=(29$ patients $\times 2$

years $)+(16$ patients $\times$ various follow-up periods $)$

In addition, 29 patients were followed up for more than 2 years after radiosurgery. Calculating the number of patient-years after the first 2 years post-GKS, we now have the following:

After the initial 2 years post-GKS: 82.4 patient-years $=(29$ patients $\times$ various follow-up periods $)-(29$ patients $\times 2$ years $)$

With the recalculation of patient-years, the annual rates of hemorrhage following GKS change as well:

Initial 2 years post-GKS: $4.29 \%=3$ hemorrhage episodes $/ 70.0$ patient-years

Please include this information when citing this paper: published online April 12, 2013; DOI: 10.3171/2013.3.GKS121066a.
After the initial 2 years post-GKS: $3.64 \%=3$ hemorrhage episodes/82.4 patient-years

These changes in percentages have been corrected throughout the article. We list the changes to the paper below; corrected changes are bolded.

Abstract, Results, Sentence 7 (page 164): "After GKS, 3 episodes of symptomatic hemorrhage were observed within the first 2 years of follow-up (4.29\% annual hemorrhage rate), and 3 episodes of symptomatic hemorrhage were observed after the first 2 years of follow-up (3.64\% annual hemorrhage rate)."

Results, Post-GKS Hemorrhage Rate, Sentences 7 and 8 (page 166): "The annual hemorrhage rate during the first 2 years after GKS was calculated to be $\mathbf{4 . 2 9 \%}$ (3 hemorrhage episodes/70.0 patient-years), and the annual hemorrhage rate after the initial 2-year followup was calculated to be $\mathbf{3 . 6 4 \%}$ (3 hemorrhage episodes/82.4 patient-years). A significant reduction ( $\mathbf{p}<$ $\mathbf{0 . 0 5}$ ) in the annual hemorrhage rate was demonstrated after GKS $(31.3 \% \rightarrow \mathbf{4 . 2 9 \%} \rightarrow \mathbf{3 . 6 4 \%})$, as shown in Fig. 2."

Discussion, Paragraph 2, Sentence 2: "In the present series of patients with brainstem CMs, the annual hemorrhage rate after GKS was $\mathbf{4 . 2 9 \%}$ for the first 2 years and 3.64\% thereafter."

Conclusions, Point 1. "Gamma Knife surgery is effective in reducing the rate of recurrent hemorrhage in patients with brainstem CMs. In this study the annual hemorrhage rate decreased from $31.3 \%$ to $\mathbf{4 . 2 9 \%}$ within 2 years and further decreased to $\mathbf{3 . 6 4 \%}$ after 2 years."

Our changes also affect Figure 2 and Table 4. The revised figure and table appear on the following page.

Although our data have changed, we still can con-

This article contains some figures that are displayed in color online but in black-and-white in the print edition. 
REVISED TABLE 4: Literature review: radiosurgical results for brainstem $\mathrm{CMs}^{*}$

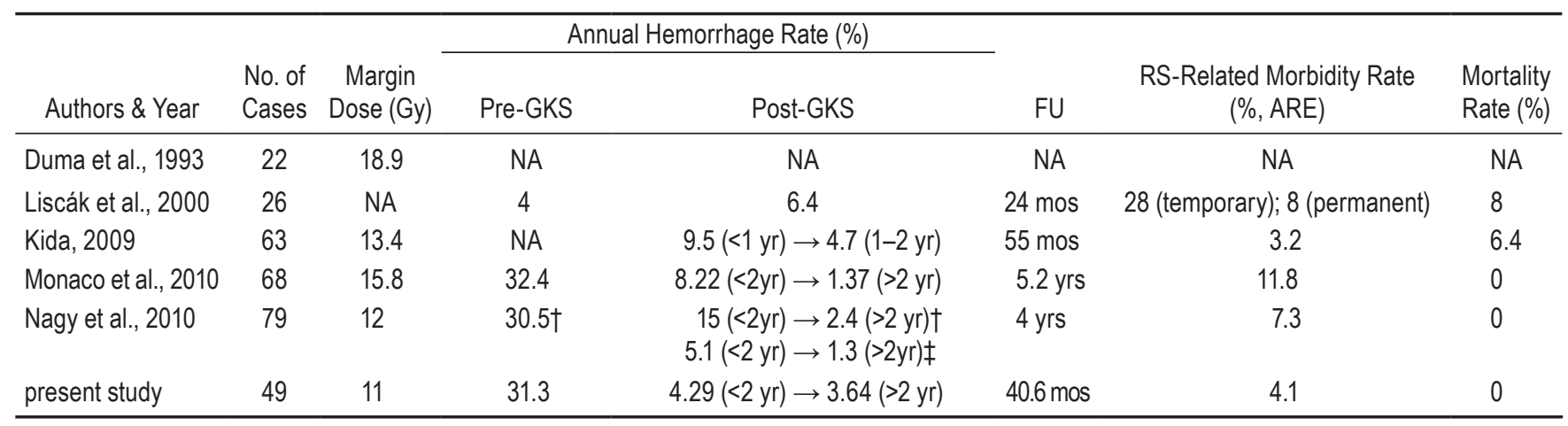

* $A R E$ = adverse radiation effect; FU = follow-up; NA = not available; $R S=$ radiosurgery.

$\dagger$ high-risk group.

$\ddagger$ low-risk group.

clude that GKS is effective in reducing the rate of recurrent hemorrhage in patients with brainstem CMs. In our study, the annual hemorrhage rate decreased from $31.3 \%$ to $4.29 \%$ within 2 years after GKS and further decreased to $3.64 \%$ after the first 2 years.

We apologize to the readers for any confusion raised by our original numbers. We thank Drs. Li and Zhang for alerting us to problems in our method of calculation, and we thank the Editor for allowing us to make our corrections at this time. The online version of the text has been corrected as of April 12, 2013.

Cheng-Chia Lee, M.D. David Hung-Chi PAN, M.D. Neurological Institute Taipei Veterans General Hospital Taipei, Taiwan

\section{Reference}

1. Li D, Zhang JT: Cavernous malformations. J Neurosurg [epub ahead of print April 12, 2013. DOI: 10.3171/2013.1.JNS1393] (Letter)

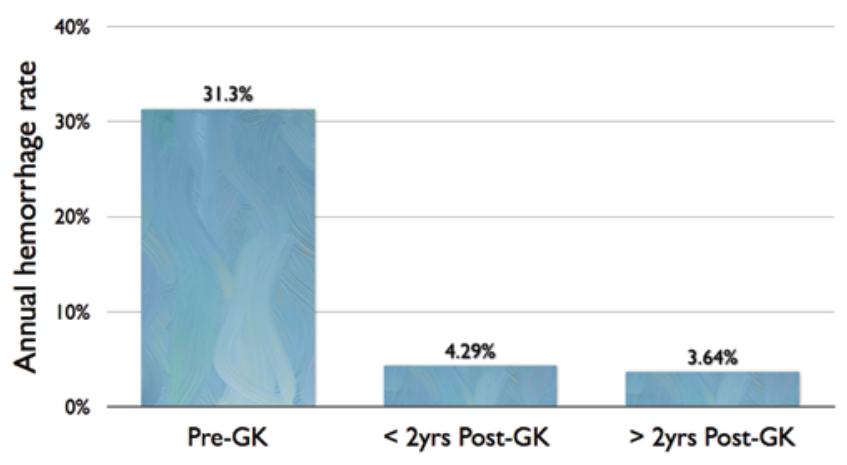

Revised Fig. 2. Bar graph demonstrating changes in the annual hemorrhage rate following GKS (within 2 years and beyond 2 years). There was a decreasing trend in the hemorrhage rate after GKS. In most patients, hemorrhage was controlled, and the patient did not suffer any further neurological deterioration. 